OPEN ACCESS

Edited by:

Eyal Grunebaum,

Hospital for Sick Children, Canada

Reviewed by:

Katrina Louise Randall,

Australian National University, Australia

Sujal Ghosh,

Heinrich Heine University of

Düsseldorf, Germany

*Correspondence:

Xiaodong Zhao

zhaoxd530@aliyun.com

Specialty section:

This article was submitted to Primary Immunodeficiencies,

a section of the journal

Frontiers in Immunology

Received: 15 April 2021 Accepted: 27 October 2021 Published: 15 November 2021

Citation:

Jiang J, Qin T, Zhang L, Liu Q, Wu J, Dai R, Zhou L, Zhao Q, Luo X, Wang H and Zhao X (2021) IL-21 Rescues the Defect of IL-10-Producing Regulatory $B$ Cells and Improves Allergic Asthma in DOCK8 Deficient Mice.

Front. Immunol. 12:695596. doi: 10.3389/fimmu.2021.695596

\section{IL-21 Rescues the Defect of IL-10-Producing Regulatory B Cells and Improves Allergic Asthma in DOCK8 Deficient Mice}

Jinqiu Jiang ${ }^{1,2}$, Tao Qin ${ }^{3}$, Liang Zhang ${ }^{2}$, Qiao Liu $^{2}$, Jiabin $W u^{2}$, Rongxin Dai ${ }^{2,4}$, Lina Zhou ${ }^{2}$, Qin Zhao ${ }^{2}$, Xiaoyan Luo ${ }^{1}$, Hua Wang ${ }^{1}$ and Xiaodong Zhao ${ }^{2,4^{*}}$

\footnotetext{
${ }^{1}$ Department of Dermatology, Ministry of Education Key Laboratory of Child Development and Disorders, National Clinical Research Center for Child Health and Disorders, China International Science and Technology Cooperation Base of Child Development and Critical Disorders, Children's Hospital of Chongqing Medical University, Chongqing, China, ${ }^{2}$ Chongqing Key Laboratory of Child Infection and Immunity, Children's Hospital of Chongqing Medical University, Chongqing, China, ${ }^{3}$ Department of Infectious Diseases, Children's Hospital of Chongqing Medical University, Chongqing, China, ${ }^{4}$ Department of Rheumatology and Immunology, Children's Hospital of Chongaing Medical University, Chongaing, China
}

Mutations in human DOCK8 cause a combined immunodeficiency syndrome characterized by allergic diseases such as asthma and food allergy. However, the underlying mechanism is unclear. Regulatory B (Breg) cells that produce IL-10 exert potent immunosuppressive functions in patients with allergic and autoimmune disorders. DOCK8-deficient B cells show diminished responses to TLR9 signaling, suggesting a possible defect in IL-10-producing Breg cells in those with DOCK8 deficiency, which may contribute to allergies. Here, we isolated peripheral blood mononuclear cells from DOCK8-deficient patients and generated a Dock8 KO mouse model to study the effect of DOCK8 deficiency on Breg cells. DOCK8-deficient patients and Dock8 KO mice harbored quantitative and qualitative defects in IL-10-producing Breg cells; these defects were caused by abnormal Dock8 ${ }^{-/-} \mathrm{CD} 4^{+} \mathrm{T}$ cells. We found that recombinant murine (rm) IL-21 restored the function of Bregs both in vitro and in Dock8 KO mice, leading to reduced inflammatory cell infiltration of the lungs in a murine asthma model. Overall, the results provide new insight into the potential design of Breg-based or IL-21-based therapeutic strategies for allergic diseases, including asthma associated with DOCK8 deficiency.

Keywords: regulatory B cells, IL-10, DOCK8 deficiency, asthma, IL-21 


\section{INTODUCTION}

Mutations in dedicator of cytokinesis 8 (DOCK8) are the major cause of autosomal recessive hyper-IgE syndromes (HIES), which are characterized by combined immunodeficiency and elevated serum IgE levels (1). Patients with HIES caused by DOCK8 deficiency are more susceptible to developing allergic diseases (e.g., asthma, food allergies, and atopic dermatitis) than those with HIES caused by STAT3 mutations (2). Recent work identified some of the mechanisms underlying the high incidence of allergy in DOCK8 deficiency. For example, Dock $8^{-1-} \mathrm{IL}-13^{\text {hi }} \mathrm{IL}-$ $4^{\text {hi }} \mathrm{IL}-5^{\text {hi }} \mathrm{IL}-21^{\text {lo }}$ follicular helper T (Tfh) 13 cells are associated with production of high-affinity IgE antibodies (3), and migrationinduced cell shattering causes a type 2-biased helper $\mathrm{T}$ cell response (4). However, the role of DOCK8-deficient B cells in the pathogenesis of allergic diseases remains unclear.

DOCK8 functions as a guanine nucleotide exchange factor that is important for actin cytoskeleton rearrangement and optimal STAT3 phosphorylation; it also serves as an adaptor molecule for TLR9-MYD88 signaling in B cells $(5,6)$. Loss-offunction mutations in DOCK8 contribute to impairment of B cell function and of long-lived memory responses (7).

$\mathrm{B}$ cells play an important role in the pathogenesis of allergic diseases, in particular by secreting IgE. However, regulatory B (Breg) cells in humans and mice are defined as B cells with immunosuppressive capacity associated with secretion of antiinflammatory cytokines such as TGF- $\beta$, IL- 35 , and, particularly, IL10 (8-11). Several Breg subsets exert immunoregulatory functions in allergic and inflammatory diseases by secreting IL-10; these cells, such as mouse $\mathrm{CD} 1 \mathrm{~d}^{\text {hi }} \mathrm{CD}^{+} \mathrm{B}$ cells (termed B10 cells) (12), CD21 ${ }^{+} \mathrm{CD} 23$ marginal zone (MZ) B cells (13), and human $\mathrm{CD} 19^{+} \mathrm{CD} 24^{\mathrm{hi}} \mathrm{CD} 27^{+}$ memory B cells (14), and $\mathrm{CD} 19^{+} \mathrm{CD} 24^{\mathrm{hi}} \mathrm{CD} 38^{\mathrm{hi}}$ immature transitional B cells (15) share partially overlapping phenotypes. IL-10-producing Breg cells have direct and indirect suppressive effects on proliferation and cytokine production by effector $\mathrm{T}$ cells. With respect to the signaling mechanisms underlying development of IL-10-producing Breg cells, previous studies report that BCR-derived signals initiate acquisition of regulatory B10-like competence. Then, LPS-induced TLR4 and TLR9 signaling facilitates a transcriptionally active conformation of the IL10 gene in pro-B10 cells $(16,17)$; also, activation of STAT3 is required for TLR-induced IL-10 production by $\mathrm{B}$ cells $(18,19)$. In addition, IL-21- and CD40-dependent cognate interactions with $\mathrm{T}$ cells are required to generate fully functional mouse B10 cells (20). DOCK8-deficient B cells show diminished responses to TLR9 signaling, suggesting a possible defect in IL-10producing Breg cells in those with DOCK8 deficiency, which may contribute to allergies.

IL-21 is a type I cytokine produced mainly by activated $\mathrm{CD} 4^{+}$ $\mathrm{T}$ cells and natural killer $\mathrm{T}$ (NKT) cells $(21,22)$. Upon binding to its receptors (IL-21R and a common receptor $\gamma$ chain), IL-21 activates the Janus family tyrosine kinases members JAK1 and JAK3, with subsequent phosphorylation of STAT3 and STAT1 (23). For functionality, IL-21 mediates maturation of B cells, normal development of $\mathrm{T}$ follicular helper cells, and differentiation of Th17 cells (24).

In this study, we generated Dock8 KO mice using the TALEN technique; these mice harbor a frameshift mutation in the first exon of Dock8, which mirrors human disease because most of the DOCK8-deficiency in patients is caused by the frame shift or gene deletion in different exons of the DOCK8 gene rather than by a point mutation. We found that DOCK8-deficient patients and Dock8 KO mice harbor both quantitative and qualitative defects in IL-10-producing Breg cells due to abnormalities in Dock $^{-1-} \mathrm{CD}^{+} \mathrm{T}$ cell populations. We also found that IL-21 rescued the function of Bregs in Dock8 KO mice and alleviated inflammatory infiltration in a murine asthma model.

\section{MATERIALS AND METHOD}

\section{Patients}

Three Chinese patients with mutations in the DOCK8 gene were enrolled in the study. All patients were admitted to the Children's Hospital of Chongqing Medical University. Diagnosis of the patients was described previously (25). Three age-matched subjects were enrolled as healthy controls (HCs). Informed consent to participate in the study was provided by the patients' families, and the study was approved by the Medical Ethics Committee of Children's Hospital of Chongqing Medical University.

\section{Mice}

Dock8 KO mice were generated using the TALEN technique (Shanghai Biomodel Organism Science \& Technology Development Co., Ltd). The first exon of Dock 8 was chosen for TALEN-induced mutagenesis; absence of a $45 \mathrm{bp}$ sequence from the coding frame of exon 1 introduced a reading frame shift into the Dock8 gene (26). Dock8 was genotyped by PCR using the following primer pair: sense, 5'- GGGGGATCCCCTGC GGCCGGCGACTCTGA-3', and antisense, 5'- GGGGAATTC GAAGCGGGGAAGGCAATGATGACA-3'. PCR products amplified from F0 generation mouse tail tissue were purified, cloned, and sequenced to identify positive founder mice with the Dock8 protein harboring the frame shift. Positive F0 generation mice were crossed with C57BL/6J mice and the genotype of the offspring was confirmed by PCR, cloning, and sequencing. CD4 $\mathrm{KO}$ and $\mathrm{CD} 45.1^{+} \mathrm{C} 57 \mathrm{BL} / 6$ mice were obtained from the Jackson Laboratory. C57BL/6 mice were purchased from the Laboratory Animal Center, Chongqing Medical University. All mice were aged 6 to 10 weeks at the time of the experiments and were housed in specific pathogen-free animal facilities. Data were obtained from three or more mice per group. All animal experiments were reviewed and approved by the Institutional Animal Care and Usage Committee of Children's Hospital of Chongqing Medical University.

\section{Flow Cytometry and Phosphorylation Analyses}

Heparinized blood was obtained from patients and from agematched HC subjects. Peripheral blood mononuclear cells (PBMCs) were isolated by Ficoll density gradient centrifugation and cell numbers were counted in a hemocytometer. Flow cytometry was performed using a FACSCanto II cytometer (BD Biosciences, San Jose, Calif). Briefly, PBMCs were stained with anti- 
human CD19-APC, anti-human CD24-PE, anti-human CD27BV450, anti-human CD38-PerCP-cy5.5, or anti-human IgDBV510. Mononuclear cells isolated from the spleen of DOCK8 $\mathrm{KO}$ and WT mice were stained with the following antibodies: antiCD19 FITC, anti-CD5 PE, and anti-CD1d APC (CD1d ${ }^{\text {hi }}$ CD5 ${ }^{+}$B cells); anti-CD19 FITC, anti-CD23 PE, anti-CD21 APC, and antiCD24 Percp-cy5.5 (T2-MZP cells) (all antibodies were from BioLegend, CA). Mononuclear cells isolated from spleen cells of chimera mice were stained with anti-CD45.1 BV510, CD45.2 FITC, anti-CD19 APC, and anti-IL-10 PE (BioLegend, CA). Mononuclear cells isolated from the spleen of CD4 KO mice were stained with anti-CD19 FITC or APC, anti-CD5 PE, anti-CD1d APC, antiCD23 PE, anti-CD21 APC, anti-CD24 Percp-cy5.5, or anti-IL-10 PE (BioLegend, CA). To detect phosphorylation, single-cell suspensions of splenocytes were stimulated for $3 \mathrm{~h}$ with lipopolysaccharide (LPS; $10 \mu \mathrm{g} / \mathrm{mL}$, Sigma, St. Louis) or for 30 min with rmIL-21 (100 ng/mL; R\&D Systems). Cells were then fixed and permeabilized with BD Phosflow Lyse/Fix Buffer and Perm Buffer III (BD Biosciences), respectively. Finally, cells were stained with anti-B220 FITC, anti-CD5 BV421, anti-CD1d APC, or anti-pY727 PE. All data were analyzed using FlowJo software.

\section{B Cell Stimulation}

PBMCs isolated from patients or HCs were resuspended (at $2 \times 10^{6}$ cells $/ \mathrm{mL}$ ) in 48 -well flat-bottom plates in culture medium and stimulated for $7 \mathrm{~h}$ with LPS $(10 \mu \mathrm{g} / \mathrm{mL}$; Sigma, St. Louis), phorbol 12-myristate 13-acetate (PMA, $20 \mathrm{ng} / \mathrm{mL}$; Sigma, St. Louis), ionomycin (1 $\mu \mathrm{g} / \mathrm{mL}$; Sigma, St. Louis), and brefeldin A (BFA, $1 \times$ solution/mL; BioLegend, CA) before staining and flow cytometry analysis. Next, the cells were harvested and washed twice with PBS. Single-cell suspensions were then stained for $20 \mathrm{~min}$ on ice with predetermined optimal concentrations of anti-CD19 APC (BioLegend, CA). Stained cells were washed twice with PBS before fixation and permeabilization in Fixation and Permeabilization Buffer (BioLegend, CA). Finally, cells were stained for 30 min with anti-IL-10 PE (BioLegend, CA). To detect B10 cells in mice, single-cell suspensions of splenocytes were stimulated for $5 \mathrm{~h}$ with LPS, PMA (50 ng/mL), ionomycin (500 ng/mL), and BFA, followed by staining with anti-CD19 APC
(BioLegend, CA) and anti-IL-10 PE (BioLegend, CA). For coculture experiments, splenic B cells were purified using an EasySep $^{\text {TM }}$ Mouse B Cell Isolation Kit (STEMCELL Technologies, Canada). The cells $\left(2 \times 10^{6}\right.$ cells $\left./ \mathrm{mL}\right)$ were then incubated for $48 \mathrm{~h}$ with mouse rmIL-21 (100 ng/mL, R\&D Systems). After culture for $48 \mathrm{~h}$, the IL-10 concentration in the supernatant was measured in an ELISA (BioLegend, CA), and B10 cell numbers were measured by flow cytometry analysis, as described above.

\section{Generation of Bone Marrow Chimeras}

Bone marrow was collected from Dock $8^{-/-}\left(\mathrm{CD} 45.2^{+}\right)$mice and from C57BL/6 wild-type $\left(\mathrm{CD} 45.1^{+}\right)$mice. For each chimera, CD 45.2 $2^{+}$ wild-type or Dock8 KO bone marrow cells plus $\mathrm{CD} 45.1^{+}$bone marrow cells $\left(4 \times 10^{6}\right.$ cells in a 1:1 mixture) were transferred intravenously into lethally-irradiated (two doses of 550 rads each) wild-type CD45. $1^{+}$recipients. Recipient mice were allowed 8 weeks to reconstitute prior to challenge with ovalbumin (OVA).

\section{Generation of OVA-Induced Allergic Asthma Model Mice and Nasal Administration of Recombinant IL-21}

OVA-induced allergic asthma was elicited by sensitization with chicken OVA (5 $\mu \mathrm{g}$, intraperitoneally (i.p.); Sigma-Aldrich) emulsified in $200 \mu \mathrm{l}$ Imject Alum (Thermo Fisher Scientific) on Day 0 , followed by two oropharyngeal aspiration challenges (on Days 14 and 21) with 1.5\% OVA dissolved in $50 \mu \mathrm{l} \mathrm{PBS}$. Control mice received only PBS. Mice were harvested $72 \mathrm{~h}$ after the second challenge (27). Some mice received $20 \mathrm{ng}$ of rmIL-21 (R\&D Systems) into the nostrils (daily on Days 15-17, 18-20, or 15-20). This 3 day protocol was decided by conducting preliminary time-course experiments. The dose of rmIL-21 was obtained from a previous study (28).

\section{Adoptive Cell Transfer}

Naïve splenic $\mathrm{CD}^{+}{ }^{+} \mathrm{T}$ cells isolated from Dock8 KO or wild-type mice were purified with the EasySep ${ }^{\mathrm{TM}}$ Mouse Naïve CD4 ${ }^{+} \mathrm{T}$ Cell Isolation Kit (STEMCELL Technologies, Canada). Next, $5 \times 10^{6}$ naïve $\mathrm{CD} 4^{+} \mathrm{T}$ cells were adoptively transferred into $\mathrm{CD} 4 \mathrm{KO}$ mice via intravenous injection 1 day before immunization with OVA.

TABLE 1 | Clinical features of DOCK8-deficient patients.

\begin{tabular}{|c|c|c|c|}
\hline Features & P1/Male & P2/Female & P3/Male \\
\hline Age at onset & $6 y$ & 1 y $7 \mathrm{~m}$ & $3 y$ \\
\hline Age at diagnosis & $14 \mathrm{y}$ & 5 y $8 \mathrm{~m}$ & $9 y$ \\
\hline DOCK8 mutation & $\begin{array}{l}\text { Exon11 homozygous deletion + Exon 12-33 } \\
\text { heterozygous deletion }\end{array}$ & $\begin{array}{c}\text { Exon2 homozygous deletion + Exon1, 3-39 } \\
\text { heterozygous deletion }\end{array}$ & $\begin{array}{l}\text { c. } 1278-1279 \text { delTG, } \\
\text { p.V427fsX435 }\end{array}$ \\
\hline $\begin{array}{l}\text { DOCK8 protein } \\
\text { expression }\end{array}$ & - & - & - \\
\hline Atopy & Eczema, asthma & Eczema, & Eczema, food allergy \\
\hline Immunodeficiency & Respiratory infection, otitis media, sinusitis, stomatitis & Respiratory infection, otitis media, stomatitis & $\begin{array}{l}\text { Respiratory infection, otitis } \\
\text { media, stomatitis }\end{array}$ \\
\hline Autoimmune disease & - & - & Autoimmune hemolytic anemia \\
\hline Malignancy & - & - & - \\
\hline Etiology of infections & HSV & EBV & EBV \\
\hline $\begin{array}{l}\text { Molluscum } \\
\text { contagiosum }\end{array}$ & + & + & + \\
\hline
\end{tabular}

HSV, herpes simplex virus; EBV, Epstein-Barr virus. ; -, negtive; +, positive. 
OVA immunization was performed as described above. $100 \mu \mathrm{l}$ blood was taken from the tail vein of CD4KO mice on day 7, day14 and day 21 to detect the number of $\mathrm{CD} 4^{+} \mathrm{T}$ cells. Recipient mice were analyzed on Day 24 post-OVA immunization.

\section{Histopathological Analysis}

Lung tissue was harvested and fixed for $24 \mathrm{~h}$ in $10 \%$ formalin and then embedded in paraffin. Sections $(4 \mu \mathrm{m})$ were stained with hematoxylin and eosin. The degree of airway infiltration by inflammatory cells was scored by two independent investigators by double-blind screening. Peri-bronchiole and peri-vascular inflammation were evaluated using a scoring system of $0-4$, where 0 represents no cells; 1, a few cells; 2, a ring of inflammatory cells one cell layer deep; 3 , a ring of inflammatory cells $2-4$ cells deep; and 4 , a ring of inflammatory cells $>4$ cells deep.

\section{Statistical Analysis}

GraphPad Prism 5 software was used for statistical analyses. Results are expressed as the mean \pm SEM. The significance of differences between groups was determined using a two tailed unpaired Student's $t$ test or ANOVA. A P value $<0.05$ was deemed statistically significant.
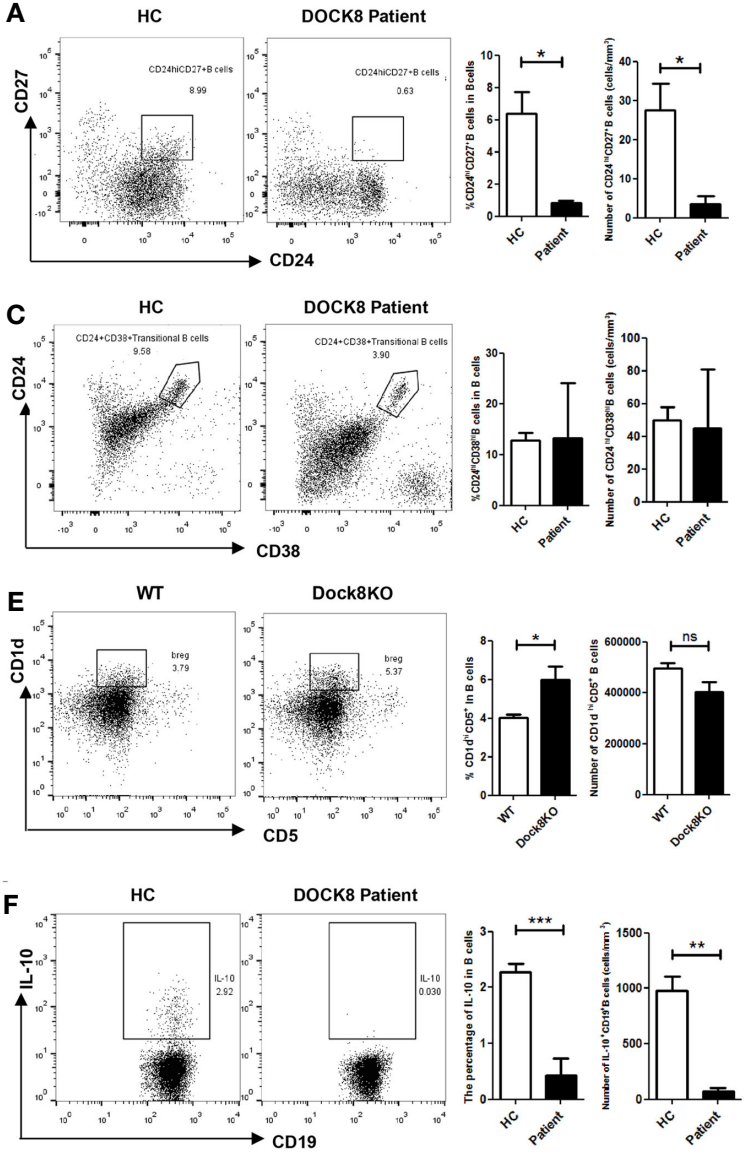

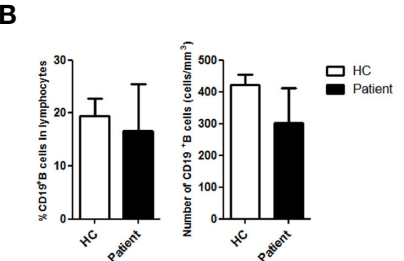

D
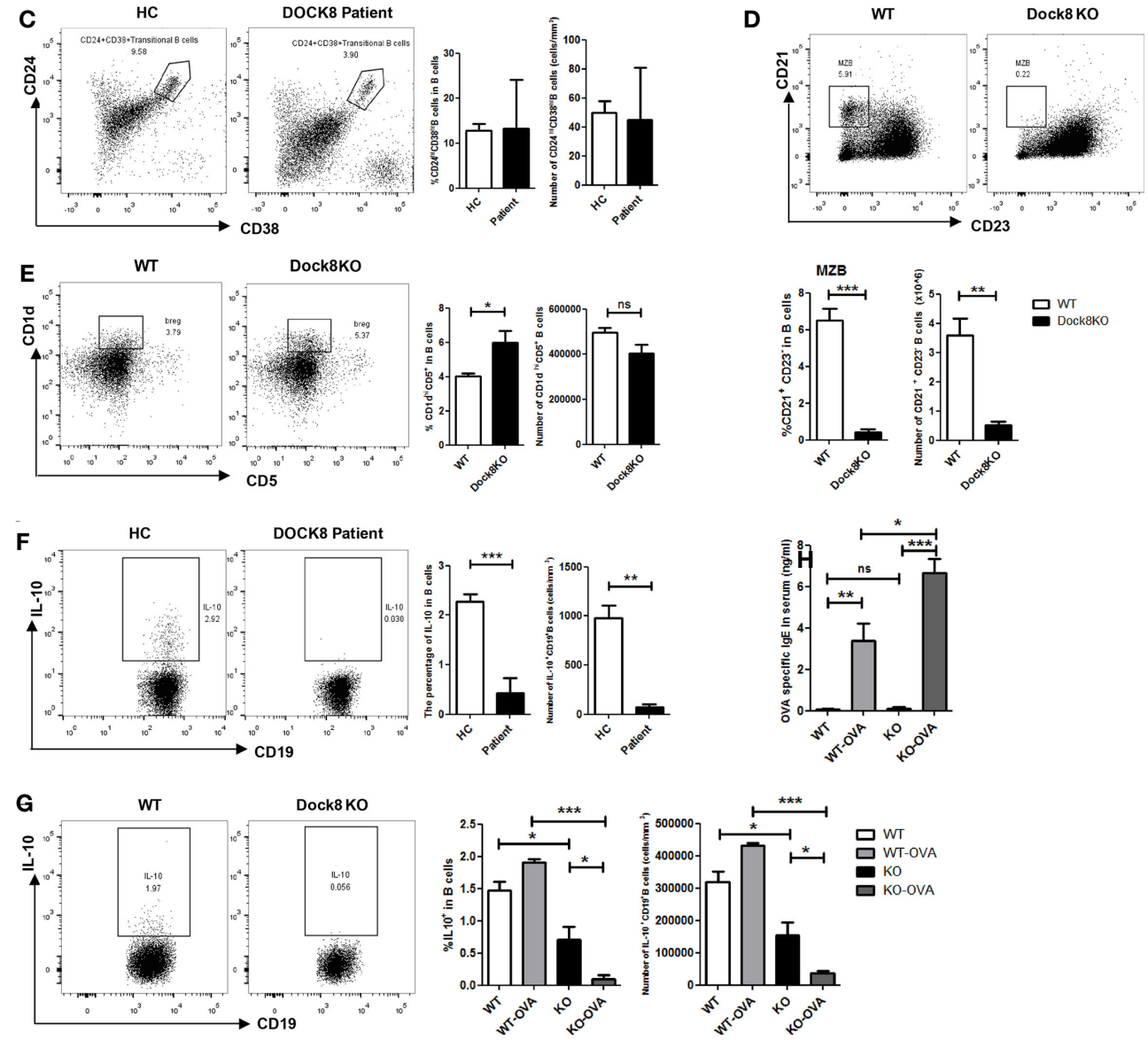

FIGURE 1 | Reduced percentage of IL-10-producing Breg cells in DOCK8-deficient patients and Dock8 KO mice. (A) Percentage and number of CD19+CD24 ${ }^{\text {hi }}$ $\mathrm{CD}_{2} 7^{+} \mathrm{B}$ cells, (B) CD19+ B cells, and (C) CD19 ${ }^{+} \mathrm{CD} 24^{\text {hi }} \mathrm{CD} 38^{\text {hi }} \mathrm{B}$ cells in HCs and DOCK8-deficient patients ( $\mathrm{n}=3 \mathrm{for}$ both). (D) CD21 ${ }^{+} \mathrm{CD} 23^{-}$marginal zone B cells and $(\mathbf{E}) \mathrm{CD}^{+} \mathrm{CD} 1 \mathrm{~d}^{\text {hi }} \mathrm{B}$ cell populations in the spleen of wild-type and Dock $8 \mathrm{KO}$ mice ( $\mathrm{n}=4$ for both). (F) The percentage of IL-10 $\mathrm{CD} 19^{+}$Breg cells in $\mathrm{HCs}$ and DOCK8-deficient patients ( $\mathrm{n}=3$ for both). (G) Percentage of IL-10+CD19+ Breg cells in the spleen of wild-type and Dock8 KO mice immunized (or not) with OVA ( $n=4$ for both). (H) OVA-specific IgE in serum of Dock8 KO mice immunized with OVA was measured by ELISA. ${ }^{*} P<0.05$, ${ }^{\star \star} P<0.01$, and ${ }^{\star \star \star} P<0.001$, and ns is not significant (Student's $t$ test). Data are representative of three independent experiments. 


\section{RESULTS}

\section{Breg Subsets Are Present in DOCK8- Deficient Patients and Dock8 KO Mice, but Are Not Capable of Producing IL-10}

To investigate the effects of DOCK8 deficiency on homeostasis of Breg cells in humans, we isolated PBMCs from three patients (aged 5-14 years) with confirmed DOCK8 deficiency (Table 1). First, we examined Breg subsets. We found that the percentage and number of $\mathrm{CD} 19^{+} \mathrm{CD} 24^{\text {hi }} \mathrm{CD} 27^{+}$memory B cells within the PBMC population was lower in patients than in age-matched HCs (Figure 1A), whereas the total B cell (Figure 1B) and $\mathrm{CD} 19^{+} \mathrm{CD} 24^{\mathrm{hi}} \mathrm{CD} 38^{\text {hi }}$ immature transitional B cell populations (Figure 1C), which harbor IL-10-producing Breg cells, were normal. In Dock8 KO mice, analysis of splenic B cells revealed a near absence of $\mathrm{CD} 21^{+} \mathrm{CD} 23^{-}$marginal zone $\mathrm{B}$ cells (Figure 1D) but a higher frequency of $\mathrm{CD} 1 \mathrm{~d}^{\text {hi }} \mathrm{CD}^{+} \mathrm{B} 10$ cells (Figure 1E) than in controls; these cell populations also harbor IL-10-producing Breg cells.

Next, we examined whether DOCK8 expression affects the function of Breg cells in DOCK8-deficient patients and Dock8 KO mice. PBMCs from patients and HCs were stimulated for $7 \mathrm{~h}$ with LPS plus PMA, ionomycin, and BFA to determine whether the absence of DOCK 8 affects IL-10 production by B cells. The results showed that $\mathrm{IL}-10^{+} \mathrm{B}$ cell numbers in patients were significantly lower than those in HCs; indeed, they were almost absent from patients (Figure 1F). The percentage and number of IL- $10^{+} \mathrm{B}$ cells in Dock8 KO mice were also lower than those in wild-type mice, especially after immunization with OVA (Figure 1G). Previously, we reported that, compared with wild-type mice, Dock8 KO mice show increased serum IgE levels and develop significant airway inflammatory infiltration and airway hyper-responsiveness in the OVA-induced allergic asthma model ${ }^{20}$, a finding that is consistent with the data presented above; the serum level of OVA-specific IgE in Dock8 KO mice was also increased (Figure 1H).

\section{DOCK8 Deficiency Causes a Partial Intrinsic Defect in Breg Cells}

To investigate whether the functional defect in Breg cells from DOCK8 KO mice is intrinsic to these cells, we transferred CD $45.2^{+}$ wild-type or DOCK8 KO bone marrow cells plus CD $45.1^{+}$bone marrow cells $\left(4 \times 10^{6}\right.$ cells in a 1:1 mixture) into lethally-irradiated (two doses of 550 rads each) wild-type CD $45.1^{+}$recipients. Recipient mice were allowed 8 weeks to reconstitute before being challenged with OVA. We found that the percentage of CD $45.2^{+} \mathrm{IL}-$ $10^{+} \mathrm{B}$ cells (Figures 2A, B) and CD $45.2^{+} \mathrm{CXCR}^{+}$Tfh cells (Supplemental Figure 1) in the spleens of immunized chimeric mice harboring Dock8-deficient bone marrow cells decreased. However, B cells in the mixed chimeras retained some IL-10producing functions (the frequency of $\mathrm{IL}-10^{+} \mathrm{B}$ cells was about $0.5 \%$, whereas that in OVA-immunized Dock8 KO mice was almost
A
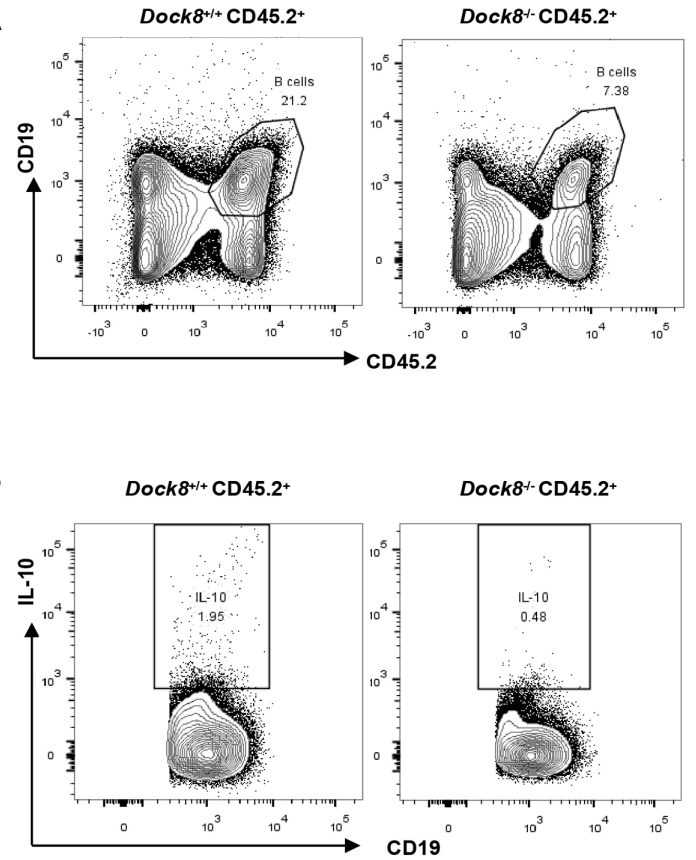
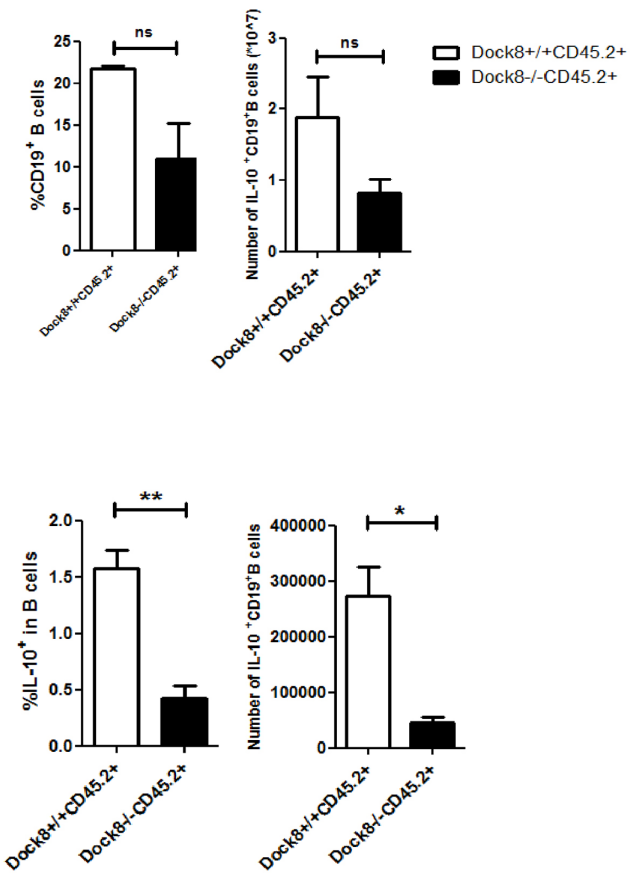

FIGURE 2 | Breg defect in Dock8 deficiency is not entirely B cell intrinsic. (A, B) CD45.2 $2^{+}$wild-type or Dock8 KO bone marrow cells were transferred intravenously into lethally-irradiated CD45. $1^{+}$wild-type recipients ( $\mathrm{n}=3$ for both) prior to immunization with OVA. Flow cytometry analysis of CD45.2 $\mathrm{CD} 19^{+} \mathrm{B}$ cells and CD45.2 ${ }^{+}$ CD19 ${ }^{+} \mathrm{L}-10^{+} \mathrm{B}$ cells in the splenocyte population from CD45.1 $1^{+}$chimeric mice. ${ }^{*} \mathrm{P}<0.05$, ${ }^{*} \mathrm{P}<0.01$, and ns is not significant (Student's $t$ test). Data are representative of two experiments. 
0\% (see Figure 1G), suggesting that IL-10 secretion by Dock8deficient $\mathrm{B}$ cells may also be affected by other cells. These results indicate that the Breg defect in Dock8 deficiency is not entirely B cell-intrinsic.

\section{DOCK8 $^{-/-}$CD4 $^{+}$T Cells Impair IL-10 Production by B Cells}

Breg cells require cognate interactions with IL-21-producing $\mathrm{CD} 4^{+}$ $\mathrm{T}$ cells to secrete IL-10 in vivo (20), and abnormalities in $\mathrm{CXCR}^{+} \mathrm{CD}^{+}{ }^{+} \mathrm{Tfh}$ cells associated with DOCK8 deficiency has been described previously (29). Therefore, we performed adoptive transfer experiments to confirm the effect of Dock $8^{-/-} \mathrm{CD} 4^{+} \mathrm{T}$ cells on IL-10 production by B cells. CD4 $\mathrm{KO}$ mice received wild-type or Dock8 KO splenic $\mathrm{CD}^{+}$naïve $\mathrm{T}$ cells, followed by challenge with OVA. The adoptively transferred $\mathrm{CD} 4^{+} \mathrm{T}$ cells in the blood of CD4 KO mice were detected on day 7, day14 and day21 (Supplemental Figure 2). At 24 days post-immunization with OVA, we analyzed the IL- $10^{+}$Breg cell population in CD4 KO recipient mice (Figure 3A). The percentage of IL- $10^{+}$Breg cells in $\mathrm{CD} 4 \mathrm{KO}$ mice receiving Dock8 $\mathrm{KO} \mathrm{CD}^{+}$naïve $\mathrm{T}$ cells was lower than that in mice receiving wild-type $\mathrm{CD} 4^{+}$naïve $\mathrm{T}$ cells (Figure 3B). There was no significant difference between two groups with respect to the percentage of $\mathrm{CD} 1 \mathrm{~d}^{\text {hi }} \mathrm{CD} 5^{+} \mathrm{B}$ cells or MZB cells (Figure 3C). Thus, Dock8 $\mathrm{KO} \mathrm{CD} 4^{+}$naïve $\mathrm{T}$ cells do not affect the percentages of Breg subsets in recipient mice. These results suggest that loss of DOCK 8 from $\mathrm{CD}^{+}{ }^{+} \mathrm{T}$ cells attenuates IL-10 production by B cells in vivo.

\section{Supplementation With IL-21 Restores IL-10 Production by B Cells From DOCK8 KO Mice Both In Vitro and In Vivo}

To confirm whether reduced IL-21 secretion by $\mathrm{CD}^{+} \mathrm{T}$ cells under conditions of Dock8 deficiency causes the functional defect in Breg cells, we co-cultured purified splenic B cells $\left(2 \times 10^{6}\right)$ from wild-type or Dock8 KO mice with IL-21. After culture for $48 \mathrm{~h}$, the IL-10 concentration in the supernatant was measured in an ELISA. Whereas the percentage of IL- $10^{+} \mathrm{B}$ cells in Dock8 KO mice was comparable with that in WT mice (Figure 4A), the concentration of IL-10 was higher (Figure 4B).

Next, to further examine the effect of IL-21 on IL-10 production by $\mathrm{B}$ cells in vivo, we exposed wild-type or Dock $8 \mathrm{KO}$ mice to intranasal rmIL-21 for 3 or 6 days. Surprisingly, the percentage of IL- $10^{+} \mathrm{B}$ cells in the spleen of Dock $8 \mathrm{KO}$ mice was almost comparable with that in wild-type mice (Figure 4C). Increased

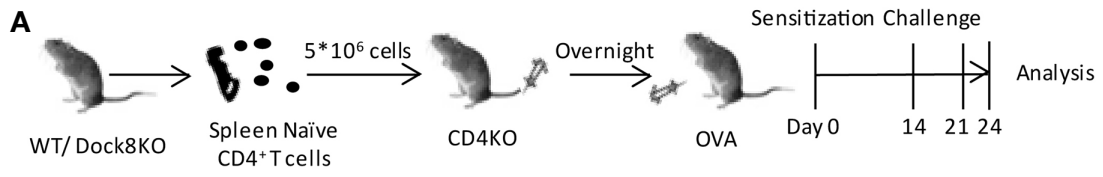

B
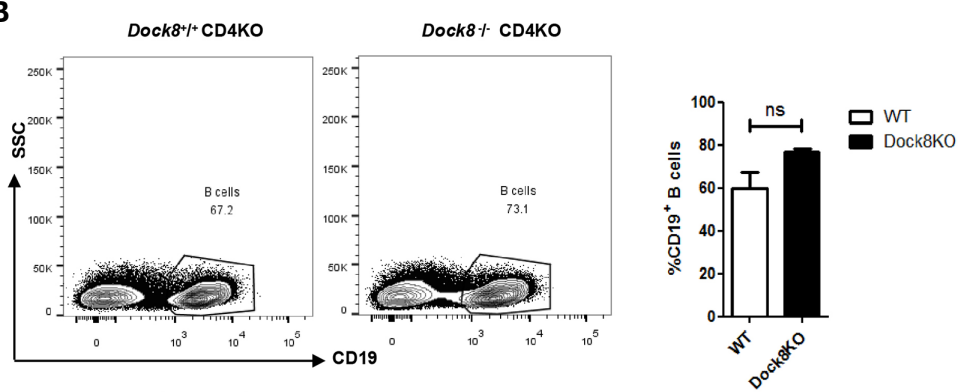

C
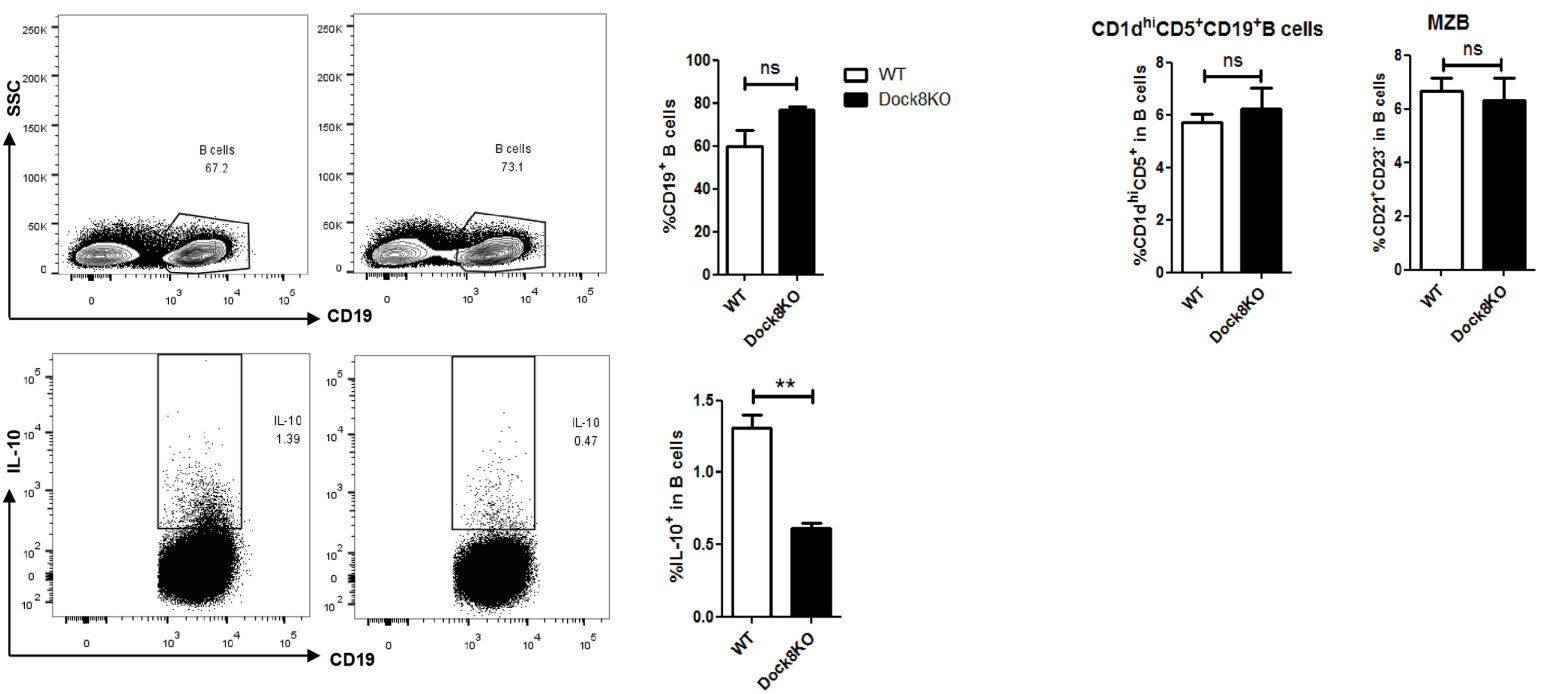

FIGURE 3 | Dock8 ${ }^{-1}$ CD4 ${ }^{+}$T cell impairs IL-10 production by B cells. (A) Splenic CD4 $4^{+}$naïve T cells from wild-type $(n=4)$ or Dock8 $\mathrm{KO}$ mice $(\mathrm{n}=4)$ were transferred into CD4 KO mice 1 day before immunization with OVA. Mice were sensitized with $5 \mu \mathrm{g}$ OVA intraperitoneally on Day 0 , followed by two oropharyngeal aspiration challenges (on Days 14 and 21). Mice were harvested $72 \mathrm{~h}$ after the second challenge. (B) The percentage of CD19+IL-10 ${ }^{+} \mathrm{B}$ cells in CD4 KO mice receiving Dock $8^{-/-} \mathrm{CD} 4^{+}$naiive T cells was lower than that in mice receiving wild-type $\mathrm{CD} 4^{+}$naiive $\mathrm{T}$ cells. (C) Dock8 ${ }^{-/-} \mathrm{CD} 4^{+} \mathrm{T}$ cells have no effect on the percentage of different Breg subtypes. ${ }^{\star \star} \mathrm{P}<0.01$, and $\mathrm{ns}$ is not significant (Student's $t$ test). Data are representative of two independent experiments. 
A
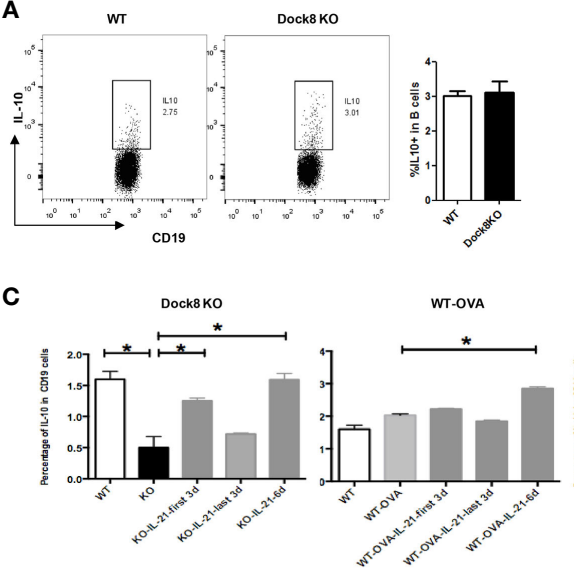

D
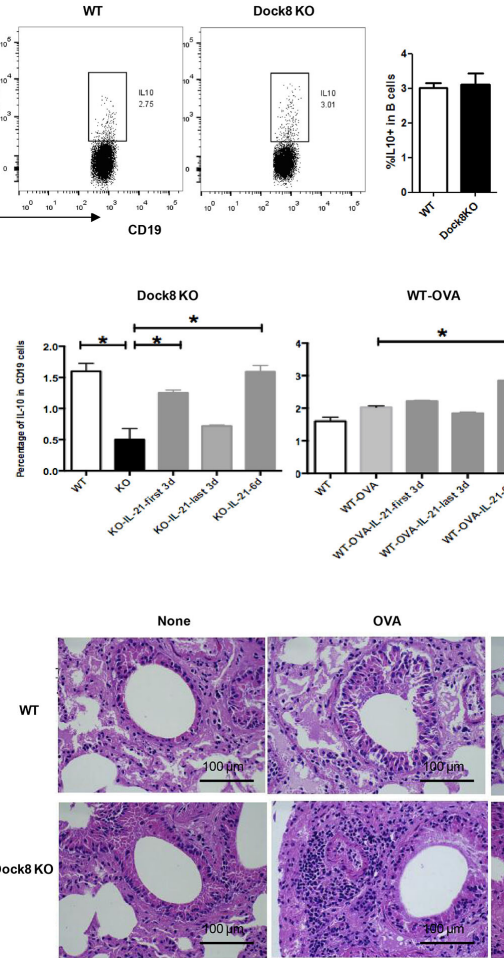

B
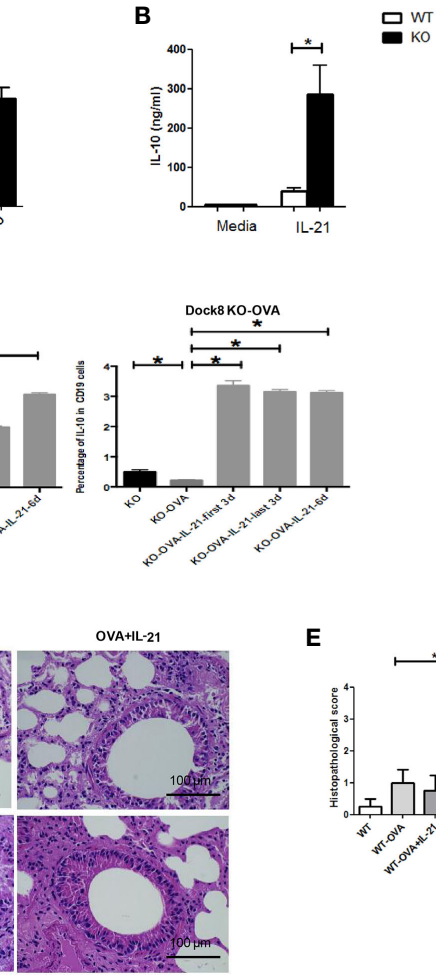

E

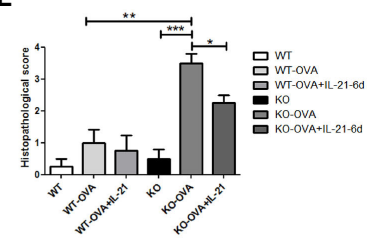

FIGURE 4 | Exogenous IL-21 rescues IL-10 production by Breg cells in Dock8 KO mice both in vitro and in vivo. (A) Splenic $\mathrm{B}$ cells isolated from wild-type ( $\mathrm{n}=3$ ) or Dock8 KO mice $(n=3)$ were co-cultured with IL-21 for 48 hours and the percentage of CD19+IL-10+ B cells was analyzed by FCM. (B) The IL-10 concentration in the supernatant was measured in an ELISA. (C) The percentage of CD19+IL-10+ $B$ cells in wild-type $(n=3)$ and Dock8 KO mice $(n=3)$, which were sensitized with $5 \mu \mathrm{g}$ OVA on Day 0, followed by two challenges (on Days 14 and 21). Finally, 20 ng of rmlL-21 was administered daily (for 3 or 6 days; Days 15-17, 18-20, or 15-20) into the nostrils. Mice were harvested $72 \mathrm{~h}$ after the second challenge. (D) Representative images of H\&E stained lung tissue from each genotype in the OVA-induced allergic asthma model."OVA+IL-21" images are from 6 days of IL-21 treatment. (E) Histopathological score for airway inflammation. ${ }^{*} P<0.05$, ${ }^{* *} P<0.01,{ }^{* *} P<0.001$, and ns is not significant (Student's $t$ test for (A, B), ANOVA for (C-E). Data in (A, B, D) are representative of three independent experiments. Data in (C) are representative of two experiments.

production of IL-10 by B cells was more obvious in Dock8 KO mice sensitized with OVA. At the same time, treatment with recombinant IL-21 provided Dock8 KO mice with marked protection from OVA-induced airway inflammation, accompanied by alleviation of inflammatory infiltration (Figures 4D, E). Whereas treatment with rmIL-21 fully restored the percentage of $\mathrm{IL}-10^{+} \mathrm{CD} 19^{+} \mathrm{B}$ cells in the spleens of Dock8 KO mice to WT levels (Figure 4C), it only partially alleviated airway inflammation (Figure 4E), suggesting that other cells might also play a role. Taken together, these data suggest that IL-21 plays a critical role in the normal function of Breg cells under conditions of Dock8 deficiency, and that exogenous IL-21 rescues defective IL-10 production by $\mathrm{B}$ cells in Dock $8 \mathrm{KO}$ mice.

\section{LPS-Driven, Not IL-21-Driven, STAT3 Phosphorylation Is Defective in Breg Cells From Dock8 KO Mice}

Because STAT3 phosphorylation is required for LPS-induced IL-10 production by B cells $(18,19)$, we examined this phenomenon in B cells from Dock8 KO mice stimulated with LPS. We found that levels of STAT3 phosphorylation in Dock8 $\mathrm{KO}$ mice were lower than those in wild-type mice (Figures 5A, B). Next, we examined STAT3 phosphorylation in CD1d ${ }^{\text {hi }} \mathrm{CD}^{+} \mathrm{B}$ cells (B10 cells) from Dock8 KO mice after stimulation with LPS or IL-21. We found no significant increase in STAT3 phosphorylation in Dock $8^{-/-}$ $\mathrm{CD} \mathrm{d}^{\text {hi }} \mathrm{CD}^{+} \mathrm{B}$ cells after LPS stimulation (Figure 5C). By contrast, IL-21 caused comparable STAT3 phosphorylation in

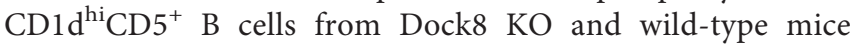
(Figures 5C, D).

\section{DISSCUSSION}

Here, we present evidence that Dock8 deficiency impairs IL-10 production by Breg cells due to abnormalities in Dock $8^{-/-}$IL-21producing $\mathrm{CD}^{+} \mathrm{T}$ cells. We also show that exogenous IL-21 rescues the function of Breg cells in Dock8 $\mathrm{KO}$ mice both in vitro and in vivo.

B cells play an important role in the pathogenesis of allergic diseases, in particular by secreting IgE. However, several 
A

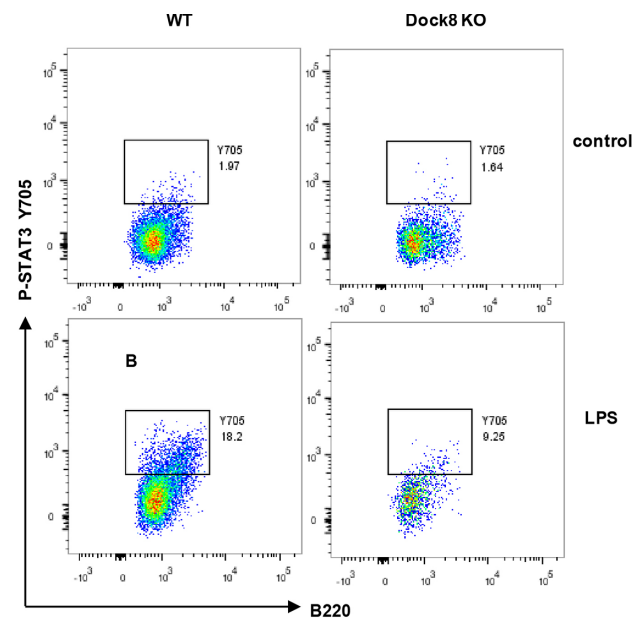

B

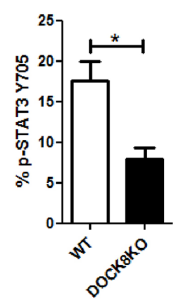

D

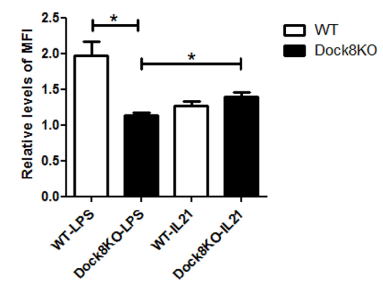

C

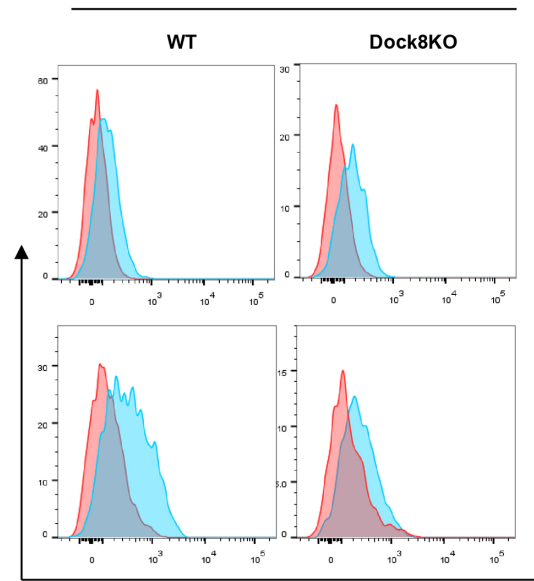

LPS
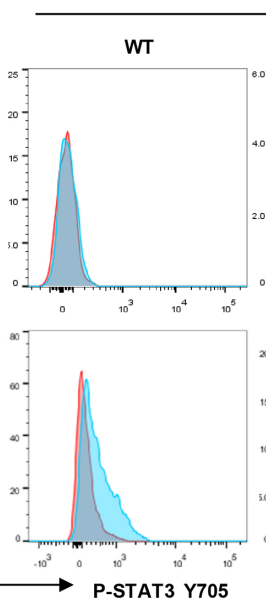

IL-21

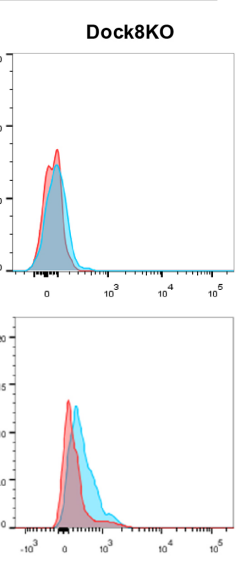

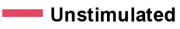

Stimulated

CD1 $\mathrm{d}^{\circ} \mathrm{CD}$-B $\mathrm{B}$ cells

CD1dhicD5+B cells

FIGURE 5 | LPS-induced STAT3 phosphorylation, but not IL-21-induced STAT3 phosphorylation, is defective in Breg cells from DOCK8 KO mice. (A, B) STAT3 phosphorylation on $Y 705$ in splenic B cells from wild-type $(n=3)$ and Dock8 KO mice $(n=3)$ after 3 hours of LPS stimulation. (C, D) STAT3 phosphorylation in $\mathrm{CD}^{+} \mathrm{CD} 1 \mathrm{~d}^{\text {hi }} \mathrm{B}$ cells and $\mathrm{CD} 5^{-} \mathrm{CD} 1 \mathrm{~d}^{\text {lo }} \mathrm{B}$ cells after stimulation with LPS for 3 hours or rmlL-21 for 30 minutes ( $\mathrm{n}=3$ for both). The red line is the unstimulated samples, the blue line is the stimulated samples. Mean fluorescence intensity (MFI) levels of IL-10 in unstimulated samples of respective groups were defined as 1. ${ }^{\star} \mathrm{P}<0.05$ (Student's $t$ test). Data in (A) are representative of three independent experiments, Data in (C) are representative of two independent experiments.

phenotypic subsets have been identified as Breg cells; these cells exert immunosuppressive functions in allergic and inflammatory diseases via release of IL-10 (30). In murine models of allergic airway disease, Breg deficiency is associated with increased serum IgE levels, increased secretion of type 2 cytokines, and increased eosinophilia $(31,32)$. Patients with allergic asthma and rhinitis show a decrease in the percentage of IL-10-secreting $\mathrm{CD} 19^{+} \mathrm{CD} 24^{\mathrm{hi}} \mathrm{CD} 27^{+}$Breg cells in response to LPS stimulation $(14,33)$. Injection of IL-10-producing $\mathrm{CD}^{+}$Breg cells into asthmatic mice normalizes airway inflammation and lung function by inhibiting Th2- and Th17-driven inflammation (34).

In the present study, we used an OVA-induced allergic asthma model based on Dock8 KO mice, as previously described (27). Model mice showed severe reductions in the percentage of IL-10-producing Breg cells, increased serum OVA-specific IgE levels, and increased inflammatory infiltration compared with wild-type mice and Dock8 KO mice not exposed to OVA. DOCK8 deficiency is a HIES; therefore, it is associated with a high incidence of allergic disease. Indeed, $71 \%$ of patients have allergic manifestations and $30 \%$ develop asthma (35). Among the three patients examined in the present study, one patient had food allergies and one had asthma. Due to the small number of enrolled patients, we were unable to test whether the number of IL-10producing Breg cells differed between patients with and without allergies. Further studies should clarify whether Breg cells are 
involved in the onset of allergic disease under conditions of DOCK8 deficiency.

Our data also provide insight into the mechanism by which DOCK8 may cause a severe reduction in Breg cell numbers. IL-10 production by Breg cells requires LPS-induced TLR signaling (36). Nonetheless, IL-21- and CD40 dependent cognate interactions with $\mathrm{T}$ cells are also required for IL-10-producing Breg cells to optimally suppress inflammation and autoimmunity (20), and adding CD40L to the LPS cultures increased IL-10 producing Bcell frequencies in the health controls (Supplemental Figure 3). DOCK8-deficient patients may also have higher numbers of IL-10 producing B-cell if stimulated appropriately. All of these signals are instrumental to Breg expansion and function; thus dysfunction leads to impaired IL-10 production and susceptibility to inflammation and allergy. Because impaired expansion of $\mathrm{CXCR}^{+} \mathrm{CD}^{+}{ }^{+} \mathrm{Tfh}$ cells has been found in DOCK8 deficiency, we adoptively transferred DOCK8 ${ }^{-/-} \mathrm{CD}^{+} \mathrm{T}$ cells into CD4 KO mice and found that this impaired IL-10 production by $\mathrm{B}$ cells, which suggests that abnormalities of Dock ${ }^{-/-} \mathrm{CD}^{+} \mathrm{T}$ cells affect the function of Breg cells. Subsequently, administration of rmIL21 improved IL-10 production by Breg cells and ameliorated airway inflammation in Dock8 KO mice. All of these results confirm that IL-21-dependent interactions with $\mathrm{T}$ cells play a critical role in the normal function of Breg cells under conditions of DOCK8 deficiency. Reduced Breg cell numbers have also been observed in a variety of allergic diseases. Whether IL- $21^{+} \mathrm{Tfh}$ cells are also involved in the defect of Breg cells remains to be explored.

As reported previously, TLR-driven STAT3 phosphorylation was defective in B cells from DOCK8-deficient patients, but IL-21 driven STAT3 phosphorylation in B cells from DOCK8-deficient patients was comparable with that in HCs (6). Our data suggest that LPS-driven, but not IL-21-driven, STAT3 phosphorylation is defective in Breg cells from Dock8 KO mice. Thus, it is possible that IL-21 restores the Breg defect in DOCK8 deficiency by inducing normal STAT3 phosphorylation.

Recently identified functions of DOCK8 explain why its loss might result in allergy; for example, generation of IgE-promoting IL-13 ${ }^{+}$follicular helper T cells (3). These "Tfh 13 " cells produce IL-13 and IL-4, but downregulate production of IL-21. Consistent with this, our data suggest that defective IL- $21^{+}$ $\mathrm{CD}^{+} \mathrm{T}$ cells might contribute to allergy in Dock8 KO mice, and that supplementation with IL-21 reduces airway inflammatory infiltration, as well as the number of serum IgE and IgE-producing B cells, in the OVA-induced allergic asthma model (27). Thus, IL-21 plays an important role in the pathogenesis of allergic asthma. However, mixed results have been reported regarding the impact of IL-21 in asthmatic mice; for example, studies suggest that IL-21 either promotes airway eosinophilia and type 2 immunity (21), or inhibits IgE production and decreases eosinophil recruitment into the airways (37). Tfh cells are the main cellular source of IL-21, which inhibits IgE class switch recombination in B cells by triggering STAT3 activation (38). Because IL-21 has a profound effect on IgE production, supplementation with IL-21 may rebalance the elevated IgE levels in patients with asthma. It is still not very clear why IL-21 signaling plays different roles in asthma; therefore, more studies are needed to provide definitive evidence.

In summary, we show here that DOCK8 regulates Breg function in both humans and mice. We propose that the absence of Bregs under conditions of DOCK8 deficiency contributes to allergy and chronic inflammation in these patients. Data from this study provide new insight into the potential design of Breg-based or IL21-based therapeutic strategies for allergic diseases, including asthma in those with DOCK8 deficiency.

\section{DATA AVAILABILITY STATEMENT}

The original contributions presented in the study are included in the article/Supplementary Material. Further inquiries can be directed to the corresponding author.

\section{ETHICS STATEMENT}

The studies involving human participants were reviewed and approved by Medical Ethics Committee of Children's Hospital of Chongqing Medical University. Written informed consent to participate in this study was provided by the participants' legal guardian/next of kin. The animal study was reviewed and approved by Medical Ethics Committee of Children's Hospital of Chongqing Medical University.

\section{AUTHOR CONTRIBUTIONS}

JJ and XZ designed the study and wrote the manuscript. JJ, TQ, LiaZ, QL, JW, RD, LinZ, QZ, XL, and HW performed the experiments and analyzed the data. TQ and XZ followed-up the patients. All authors contributed to the article and approved the submitted version.

\section{FUNDING}

This work was supported by the National Natural Science Foundation of China (81803140).

\section{ACKNOWLEDGMENTS}

We are grateful to the patients and their families for their cooperation with this study. We thank the members of the laboratory for technical assistance.

\section{SUPPLEMENTARY MATERIAL}

The Supplementary Material for this article can be found online at: https://www.frontiersin.org/articles/10.3389/fimmu.2021. 695596/full\#supplementary-material 


\section{REFERENCES}

1. Zhang Q, Jing H, Su HC. Recent Advances in DOCK8 Immunodeficiency Syndrome. J Clin Immunol (2016) 36(5):441-9. doi: 10.1007/s10875-0160296-z

2. Ponsford MJ, Klocperk A, Pulvirenti F, Dalm V, Milota T, Cinetto F, et al. Hyper-IgE in the Allergy Clinic-When is it Primary Immunodeficiency? Allergy (2018) 73(11):2122-36. doi: 10.1111/all.13578

3. Gowthaman U, Chen JS. Identification of a T Follicular Helper Cell Subset That Drives Anaphylactic IgE. Science (2019) 365(6456):eaaw6433. doi: $10.1126 /$ science.aaw6433

4. Schneider C, Shen C, Gopal AA, Douglas T, Forestell B, Kauffman KD, et al. Migration-Induced Cell Shattering Due to DOCK8 Deficiency Causes a Type 2-Biased Helper T Cell Response. Nat Immunol (2020) 21(12):1528-39. doi: 10.1038/s41590-020-0795-1

5. Keles S, Charbonnier LM, Kabaleeswaran V, Reisli I, Genel F, Gulez N, et al. Dedicator of Cytokinesis 8 Regulates Signal Transducer and Activator of Transcription 3 Activation and Promotes TH17 Cell Differentiation. J Allergy Clin Immunol (2016) 138(5):1384-94.e2. doi: 10.1016/j.jaci.2016.04.023

6. Jabara HH, McDonald DR, Janssen E, Massaad MJ, Ramesh N, Borzutzky A, et al. DOCK8 Functions as an Adaptor That Links TLR-MyD88 Signaling to B Cell Activation. Nat Immunol (2012) 13(6):612-20. doi: $10.1038 /$ ni.2305

7. Randall KL, Lambe T, Johnson AL, Treanor B, Kucharska E, Domaschenz H, et al. Dock8 Mutations Cripple B Cell Immunological Synapses, Germinal Centers and Long-Lived Antibody Production. Nat Immunol (2009) 10 (12):1283-91. doi: 10.1038/ni.1820

8. Shen P, Roch T, Lampropoulou V, O'Connor RA, Stervbo U, Hilgenberg E, et al. IL-35-Producing B Cells are Critical Regulators of Immunity During Autoimmune and Infectious Diseases. Nature (2014) 507(7492):366-70. doi: 10.1038 /nature12979

9. Parekh VV, Prasad DVR, Banerjee PP, Joshi BN, Kumar A, Mishra GC. B Cells Activated by Lipopolysaccharide, But Not By Anti-Ig and Anti-CD40 Antibody, Induce Anergy in CD8+ T Cells: Role of TGF-?1. J Immunol (2003) 170(12):5897-911. doi: 10.4049/jimmunol.170.12.5897

10. Carter NA, Vasconcellos R, Rosser EC, Tulone C, Muoz-Suano A, Kamanaka $\mathrm{M}$, et al. Mice Lacking Endogenous IL-10-Producing Regulatory B Cells Develop Exacerbated Disease and Present With an Increased Frequency of Th1/Th17 But a Decrease in Regulatory T Cells. J Immunol (2011) 186 (10):5569. doi: 10.4049/jimmunol.1100284

11. Iwata Y, Matsushita T, Horikawa M, Dilillo DJ, Yanaba K, Venturi GM, et al. Characterization of a Rare IL-10-Competent B-Cell Subset in Humans That Parallels Mouse Regulatory B10 Cells. Blood (2011) 117(2):530. doi: 10.1182/ blood-2010-07-294249

12. Yanaba K, Bouaziz JD, Haas KM, Poe JC, Fujimoto M, Tedder TF. A Regulatory B Cell Subset With a Unique CD1dhiCD5+ Phenotype Controls T Cell-Dependent Inflammatory Responses. Immunity (2008) 28(5):639-50. doi: 10.1016/j.immuni.2008.03.017

13. Bankoti R, Gupta K, Levchenko A, Stager S. Marginal Zone B Cells Regulate Antigen-Specific T Cell Responses During Infection. J Immunol (2012) 188 (8):3961-71. doi: 10.4049/jimmunol.1102880

14. Le VDV, Mlejnek E, Ozir-Fazalalikhan A, Janssen BM, Dijksman TR, Labuda LA, et al. CD24(hi)CD27(+) B Cells From Patients With Allergic Asthma Have Impaired Regulatory Activity in Response to Lipopolysaccharide. Clin Exp Allergy J Br Soc Allergy Clin Immunol (2014) 44(4):517-28. doi: 10.1111/ cea.12238

15. Blair PA, Norena LY, Flores-Borja F, Rawlings DJ, Isenberg DA, Ehrenstein MR, et al. CD19(+)CD24(hi)CD38(hi) B Cells Exhibit Regulatory Capacity in Healthy Individuals But are Functionally Impaired in Systemic Lupus Erythematosus Patients. Immunity (2010) 32(1):129-40. doi: 10.1016/ j.immuni.2009.11.009

16. Yanaba K, Bouaziz JD, Matsushita T, Tsubata T, Tedder TF. The Development and Function of Regulatory B Cells Expressing IL-10 (B10 Cells) Requires Antigen Receptor Diversity and TLR Signals. J Immunol (2009) 182(12):7459-72. doi: 10.4049/jimmunol.0900270

17. Candando KM, Lykken JM, Tedder TF. B10 Cell Regulation of Health and Disease. Immunol Rev (2014) 259(1):259-72. doi: 10.1111/imr.12176
18. Liu BS, Cao Y, Huizinga TW, Hafler DA, Toes RE. TLR-Mediated STAT3 and ERK Activation Controls IL-10 Secretion by Human B Cells. Eur J Immunol (2014) 44(7):2121-9. doi: 10.1002/eji.201344341

19. Banko Z, Pozsgay J, Szili D, Toth M, Gati T, Nagy G, et al. Induction and Differentiation of IL-10-Producing Regulatory B Cells From Healthy Blood Donors and Rheumatoid Arthritis Patients. J Immunol (2017) 198(4):151220. doi: 10.4049/jimmunol.1600218

20. Yoshizaki A, Miyagaki T, DiLillo DJ, Matsushita T, Horikawa M, Kountikov EI, et al. Regulatory B Cells Control T-Cell Autoimmunity Through IL-21Dependent Cognate Interactions. Nature (2012) 491(7423):264-8. doi: 10.1038/nature11501

21. Coquet JM, Schuijs MJ, Smyth MJ, Deswarte K, Beyaert R, Braun H, et al. Interleukin-21-Producing CD4(+) T Cells Promote Type 2 Immunity to House Dust Mites. Immunity (2015) 43(2):318-30. doi: 10.1016/ j.immuni.2015.07.015

22. Parrish-Novak J. Interleukin 21 and its Receptor are Involved in NK Cell Expansion and Regulation of Lymphocyte Function. Nature (2000) 408 (6808):57-63. doi: 10.1038/35040504

23. Novy P, Huang X, Leonard WJ, Yang Y. Intrinsic IL-21 Signaling Is Critical for CD8 T Cell Survival and Memory Formation in Response to Vaccinia Viral Infection. J Immunol (2011) 186(5):2729-38. doi: 10.4049/jimmunol.1003009

24. Habib T, Nelson A, Kaushansky K. IL-21: A Novel IL-2-Family Lymphokine That Modulates B, T, and Natural Killer Cell Responses. J Allergy Clin Immunol (2003) 112(6):1033-45. doi: 10.1016/j.jaci.2003.08.039

25. Qin T, An Y, Liu C, Wu J, Dai R, Liu D, et al. Novel DOCK8 Gene Mutations Lead to Absence of Protein Expression in Patients With Hyper-IgE Syndrome. Immunol Res (2016) 64(1):260-71. doi: 10.1007/s12026-015-8745-y

26. Sun X, Wang J, Qin T, Zhang Y, Liu C. Dock8 Regulates BCR Signaling and Activation of Memory B Cells via WASP and CD19. Blood Adv (2018) 2 (4):401. doi: 10.1182/bloodadvances.2017007880

27. Wu J, Zhang S, Qin T, Jiang J, Liu Q, Zhang L, et al. IL-21 Alleviates Allergic Asthma in DOCK8-Knockout Mice. Biochem Biophys Res Commun (2018) 501(1):92-9. doi: 10.1016/j.bbrc.2018.04.179

28. Hiromura Y, Kishida T, Nakano H, Hama T, Imanishi J, Hisa Y, et al. IL-21 Administration Into the Nostril Alleviates Murine Allergic Rhinitis. J Immunol (2007) 179(10):7157-65. doi: 10.4049/jimmunol.179.10.7157

29. Krishnaswamy JK, Gowthaman U, Zhang B, Mattsson J, Szeponik L, Liu D, et al. Migratory CD11b+ Conventional Dendritic Cells Induce T Follicular Helper Cell-Dependent Antibody Responses. Sci Immunol (2017) 2(18): eaam9169. doi: 10.1126/sciimmunol.aam9169

30. Willem VDV, Stanic B, Wirz OF, Jansen K, Globinska A, Akdis M. Role of Regulatory B Cells in Immune Tolerance to Allergens and Beyond. J Allergy Clin Immunol (2016) 138(3):654-65. doi: 10.1016/j.jaci.2016.07.006

31. Amu S, Saunders SP, Kronenberg M, Mangan NE, Atzberger A, Fallon PG. Regulatory B Cells Prevent and Reverse Allergic Airway Inflammation via FoxP3-Positive T Regulatory Cells in a Murine Model. J Allergy Clin Immunol (2010) 125(5):1114-2147483647. doi: 10.1016/j.jaci.2010.01.018

32. Singh A, Carson WF, Secor ER, Guernsey LA, Flavell RA, Clark RB, et al. Regulatory Role of B Cells in a Murine Model of Allergic Airway Disease. J Immunol (2008) 180(11):7318-26. doi: 10.4049/jimmunol.180.11.7318

33. Salomon S, Guignant C, Morel P, Flahaut G, Brault C, Gourguechon C, et al. Th17 and CD24hiCD27+ Regulatory B Lymphocytes are Biomarkers of Response to Biologics in Rheumatoid Arthritis. Arthritis Res Ther (2017) 19 (1):33. doi: 10.1186/s13075-017-1244-x

34. Braza F, Chesne J, Durand M, Dirou S, Brosseau C, Mahay G, et al. A Regulatory CD9+B-Cell Subset Inhibits HDM-Induced Allergic Airway Inflammation. Allergy (2015) 70(11):1421-31. doi: 10.1111/all.12697

35. Aydin SE, Kilic SS, Aytekin C, Kumar A, Porras O, Kainulainen L, et al. DOCK8 Deficiency: Clinical and Immunological Phenotype and Treatment Options - a Review of 136 Patients. J Clin Immunol (2015) 35(2):189-98. doi: 10.1007/s10875-014-0126-0

36. Lampropoulou V, Hoehlig K, Roch T, Neves P, Gomez EC, Sweenie CH, et al. TLR-Activated B Cells Suppress T Cell-Mediated Autoimmunity. J Immunol (2008) 180(7):4763-73. doi: 10.4049/jimmunol.180.7.4763

37. Suto A, Nakajima H, Hirose K, Suzuki K, Kagami S, Seto Y, et al. Interleukin 21 Prevents Antigen-Induced IgE Production by Inhibiting Germ Line C (epsilon) Transcription of IL-4-Stimulated B Cells. Blood (2002) 100 (13):4565-73. doi: 10.1182/blood-2002-04-1115 
38. Yang Z, Wu CM, Targ S, Allen CDC. IL-21 is a Broad Negative Regulator of IgE Class Switch Recombination in Mouse and Human B Cells. J Exp Med (2020) 217(5):e20190472. doi: 10.1084/ jem.20190472

Conflict of Interest: The authors declare that the research was conducted in the absence of any commercial or financial relationships that could be construed as a potential conflict of interest.

Publisher's Note: All claims expressed in this article are solely those of the authors and do not necessarily represent those of their affiliated organizations, or those of the publisher, the editors and the reviewers. Any product that may be evaluated in this article, or claim that may be made by its manufacturer, is not guaranteed or endorsed by the publisher.

Copyright (c) 2021 Jiang, Qin, Zhang, Liu, Wu, Dai, Zhou, Zhao, Luo, Wang and Zhao. This is an open-access article distributed under the terms of the Creative Commons Attribution License (CC BY). The use, distribution or reproduction in other forums is permitted, provided the original author(s) and the copyright owner(s) are credited and that the original publication in this journal is cited, in accordance with accepted academic practice. No use, distribution or reproduction is permitted which does not comply with these terms. 